\title{
Influence of COVID-19 Related Quarantine on Thyroid Function and Depression in Egyptian Patients with Overt Hypothyroidism
}

\author{
Ahmed Abd El-Hakim Arafat ${ }^{1}$, Hanan Abdelhay Elsherbiny ${ }^{1}$, Shady \\ Ibrahim Mashaly ${ }^{2}$, Mohammed Youssef Elshaer ${ }^{3}$, Mohammed Ali Gameil ${ }^{1}$ \\ ${ }^{1}$ Department of Internal Medicine, Faculty of Medicine, Mansoura University, \\ Mansoura, Dakahlia, Egypt. \\ ${ }^{2}$ Department of Psychiatry, Faculty of Medicine, Mansoura University, \\ Mansoura, Dakahlia, Egypt. \\ ${ }^{3}$ Department of Clinical Pathology, Faculty of Medicine, Mansoura University, \\ Mansoura, Dakahlia, Egypt.
}

Corresponding Author Mohammed Ali Gameil

Mobile:

00201099975071

E mail:

drmaligameil1979@ya hoo.com

dr_maligameil79@ma ns.edu.eg

Key words: COVID-19;

Quarantine; Hypothyroidism; Depression, $P H Q-9$ scale
Background and study aim: Currently, the coronavirus disease 2019 (COVID-19) pandemic represents a major threat to the health and social life of human beings. National quarantine was one of the tools to manage the dramatic increased spread and deaths of COVID-19. We aimed to detect the alterations of thyroid function and psychological consequences among Egyptian patients with hypothyroidism during COVID-19 related quarantine.

Patients and Method: A longitudinal observational study was conducted at the endocrine outpatient clinic of Mansoura University Hospital, Egypt during the period from March to August 2020. 160 Egyptian patients with pre-existing primary hypothyroidism were included. Patients with properly compensated thyroid function and without depression symptoms were enrolled. Patients with proven, suspected COVID-19 or prior

\section{INTRODUCTION}

Currently, the corona virus disease2019 (COVID-19) pandemic seriously threatens the health of human being with increasing deaths. Unfortunately, the pandemic of COVID-19 adversely affected mental and physical health of the general population [1]. High transmissibility, mortality rate, diverse course, lack of approved treatment, limited tools of medical aids, lockdown related restricted movement and social isolation resulted in detrimental effects on the psychological health of the public [2]. depression were excluded. After ethical approval and consent, sequential followup visits in March, June, and August 2020 were scheduled. Clinical history, examination, and laboratory assessment of thyroid function were applied regularly. Depression was evaluated via the Patient Health Questionnaire-9 (PHQ9) scale.

Results: Among 160 participants with a mean age of 42.34 and a female proportion of $70.6 \%$, TSH was positively correlated with the grade of depression and PHQ-9 score $(\mathrm{p}<0.001)$. We noticed a progressively increased prevalence of mild depression; from 3.8\% (June 2020) to $14.4 \%$ (August 2020).

Conclusion: During the lockdown period of COVID-19, Egyptian patients with preexisting hypothyroidism experienced a progressively increased prevalence of mild depression .

In March 2020 the Egyptian government imposed a national lockdown to control the dramatic transmission of COVID-19 and increased death rate. Commonly, restriction measures of the lockdown are associated with an unpleasant sensation, and multiple adverse psychological effects. Stress resulted from fear of infection, death, limitations of social activities, work and restricted movement adversely affected the psychological health of the general population [3-5]. The endocrine system; particularly adrenal and thyroid provide physiological 
changes to cope with the stressful situations [6, 7]. Hypothyroidism is considered the most prevalent endocrine disorder affecting more than $10 \%$ of humans globally [8],

A peculiar interrelationship between depression and thyroid dysfunction has been identified. Depression may develop in more than half of the patients with hypothyroidism. Moreover, disrupted thyroid function is usually encountered in patients with depression [9]. Commonly, thyroid disorders particularly autoimmune thyroiditis with elevated thyroid autoantibodies coincide with depression. The risk of depression is increased more than 6 times in patients with thyroid disorders than others [10 - 13].

We aimed to detect the prevalence and grade of depression as well as alterations of thyroid function among Egyptian patients with preexisting hypothyroidism throughout the lockdown period of COVID-19.

\section{PATIENTS AND METHODS}

An observational longitudinal follow-up study was conducted at the endocrine outpatient clinic of Mansoura University Hospital, Egypt during the period from March 2020 to August 2020. This study included 160 Egyptian patients with pre-existing hypothyroidism, aged 18-70 years, who attended their scheduled follow-up visits during the lockdown period of COVID-19. Primary hypothyroidism and Hashimoto's thyroiditis were the main causes of hypothyroidism of the participants. Participants with proper adherence to levothyroxine replacement therapy and proper compensation were included. They were subjected to a comprehensive education for the proper dosage and time of levothyroxine administration on empty stomach; at least one hour before breakfast. During the Holy Ramadan (April/May 2020) the patients were instructed to administer levothyroxine one hour before Suhor (pre-dawn meal). Recorded characteristics of the patients in December 2019 were considered as baseline data at the pre-quarantine visit. We excluded patients out of age limits, dropped-out patients, reluctant patients for thyroid function monitoring, poor adherence, prior history of thyroid surgery or radioactive iodine ablation therapy, pregnant, patients with previous or current; proven or suspected COVID-19 exposure and patients with prior depression symptoms as shown in figure
[1]. Moreover, any patient who acquired COVID-19 infection during the study was excluded. We excluded patients with acute or chronic co-morbidities such as cardiac, renal, hepatic, pulmonary diseases, systemic infections, cancer, other endocrine disorders, other autoimmune diseases or administrating medications that my affect the study outcomes such as glucocorticoid, dopamine agonists, somatostatin analogues, amiodarone, metformin, lithium, tricyclic antidepressants, selective serotonin reuptake inhibitors, antiepileptic drugs, interferon-alpha, cytotoxic, antineoplastic, biological therapy, immune therapy or iodinebased contrast exposure.

Follow-up visits were scheduled at the beginning of COVID-19 lockdown (March 2020), after three months (June 2020), and after five months (August 2020). These visits were electronically arranged to avoid overcrowding as part of the national measures to minimize the risk of infection transmission. All cases were subjected to a detailed history taking, physical examination with anthropometric measures, and laboratory testing of thyroid stimulating hormone (TSH). In every follow-up visit, about $3 \mathrm{ml}$ of venous blood was withdrawn for assessment of TSH using immune-luminescent method (Abbott i2000, Wiesbaden, Germany) with intra-assay coefficient of variation (CV) of $3.10 \%$ and an inter-assay $\mathrm{CV}$ of $3.50 \%$. TSH for normal population range is $0.4-4.2 \mathrm{mIU} / \mathrm{l}$. Ultrasensitive TSH assessment represent a corner stone for evaluation of thyroid disorders. TSH is evident to predict slight disruption of thyroid function. It is more sensitive than serum based thyroid hormone in anticipation of thyroid dysfunction. [14, 15]. Unlike ultrasensitive TSH, total and free thyroxin levels were not available for the pre-quarantine visit; December 2019 for some participants, therefore were ruled out from statistical work. Following the onset of the quarantine, the prevalence and grade of depression was evaluated for all participants based on the Patient Health Questionnaire-9 (PHQ-9) scale by the psychiatrist co-investigator. It is a 9-item depression module quoted from the full PHQ. The questionnaire includes 9 questions, and the answer of each question ranges between 0 and 3 . Thus, zero is the lower limit while 27 is the major limit for PHQ-9. Patients were diagnosed with minimal or no depression on having $0-4$ points, mild depression $5-9$ points, moderate depression 10 
- 14 points, and higher scores for moderately severe and severe depression [16].

\section{Statistical analysis}

IBM's SPSS v.23 was used for statistical analysis of the collected data. The Shapiro-Wilk test was used to check the normality of the data distribution. P-value $<0.05$ was considered statistically significant. Quantitative variables were expressed as mean and standard deviation, median, inter-quartile range and minimum to maximum range while categorical variables were expressed as frequency and percentage. Values of the Follow-up visits were compared to their corresponding baseline values using paired-t test and Wilcoxon matched pairs signed ranks test. Pearson's and Spearman's correlation coefficient tests were applied.

\section{RESULTS}

This study included 160 patients with a mean age 42.34 year and female proportion 70.6\%.The mean duration of hypothyroidism was $26.06 \pm 10.095$ months with a mean daily levothyroxine dose $89.06 \pm 24.713 \mathrm{mcg}$. Regarding residence; 103(64.4\%) cases were from rural areas and 57(35.6\%) were urban residents as shown in table (1).

Table (2) shows the progress of TSH level in the sequential follow-up visits during COVID-19 lockdown. TSH showed slight changes, but with statistically significant differences in June and August 2020 compared to December 2019 with mean values of $2.54 \pm 1.313$ and $2.73 \pm 1.425$ $\mathrm{mIU} / \mathrm{L}$ versus $2.38 \pm 1.216$, respectively ( $\mathrm{p}<$ $0.001)$. However, non-significant difference was observed in March 2020; the onset of the lockdown $(\mathrm{p}=0.287)$. (See table 2$)$

Table (3) shows the prevalence and grade of depression among the participants during the subsequent follow-up visits. With use of PHQ-9 scale, the prevalence of mild depression increased progressively from $3.8 \%$ (June 2020) to $14.4 \%$ in (August 2020)

TSH was positively correlated with PHQ-9 score at June and August $2020(\mathrm{r}=0.699$ and 0.940, respectively; $\mathrm{p}<0.001$ ). Also, a significant positive correlation was noticed between TSH and grade of depression in June and August 2020 $(\mathrm{r}=0.441$ and 0.781 , respectively; $\mathrm{p}<0.001)$ as shown in table (4).

Table (1): Demographic baseline characteristics of the participants.

\begin{tabular}{|c|c|c|c|c|c|}
\hline \multicolumn{2}{|l|}{ All patients $(n=160)$} & Mean \pm SD & Median & Range & IQR \\
\hline \multicolumn{2}{|l|}{ Age (years) } & $42.34 \pm 11.861$ & 43.00 & $19.00,68.00$ & $33.25,50.00$ \\
\hline \multicolumn{2}{|c|}{ Hypothyroidism duration (months) } & $26.06 \pm 10.095$ & 27.00 & $6.00,42.00$ & $18.00,36.00$ \\
\hline \multicolumn{2}{|c|}{ Levothyroxine dose (mcg) } & $89.06 \pm 24.713$ & 100.00 & $50.00,150.00$ & $75.00,100.00$ \\
\hline \multirow{2}{*}{ Gender (\%,NO) } & Male & \multicolumn{4}{|c|}{$29.4 \%(47)$} \\
\hline & Female & \multicolumn{4}{|c|}{$70.6 \%(113)$} \\
\hline \multirow{2}{*}{ Residence( \%, NO) } & Urban & \multicolumn{4}{|c|}{$35.6 \%(57)$} \\
\hline & Rural & \multicolumn{4}{|c|}{$64.4 \%(103)$} \\
\hline
\end{tabular}

Table (2): Sequential changes of TSH during the follow-up visits.

\begin{tabular}{|l|c|c|c|c|c|c|}
\hline \multicolumn{1}{|c|}{ All patients $(\mathbf{n}=\mathbf{1 6 0})$} & Mean \pm SD & Median & Range & IQR & 95\% CI & P \\
\hline December 2019 & $2.38 \pm 1.216$ & 2.33 & $0.43,4.71$ & $1.19,3.39$ & - & - \\
\hline March 2020 & $2.37 \pm 1.212$ & 2.38 & $0.43,5.13$ & $1.26,3.41$ & $-0.01,0.04$ & 0.287 \\
\hline June 2020 & $2.54 \pm 1.313$ & 2.56 & $0.51,5.44$ & $1.31,3.61$ & $-0.20,-0.12$ & $<\mathbf{0 . 0 0 1 *}$ \\
\hline August 2020 & $2.73 \pm 1.425$ & 2.67 & $0.53,6.42$ & $1.34,3.84$ & $-0.41,-0.29$ & $<\mathbf{0 . 0 0 1 *}$ \\
\hline
\end{tabular}

*Statistically significant $(\mathrm{p}<0.05)$ 
Table (3): Prevalence and grade of depression with PHQ-9 scale during the follow-up visits.

\begin{tabular}{|c|c|c|c|c|c|c|}
\hline & \multicolumn{2}{|c|}{ All patients $(n=160)$} & Mean \pm SD & Median & Range & IQR \\
\hline \multirow[t]{2}{*}{ Score } & \multicolumn{2}{|c|}{$\begin{array}{l}\text { June } 2020 \\
\end{array}$} & $1.29 \pm 1.338$ & 1.00 & $0.00,7.00$ & $0.00,2.00$ \\
\hline & \multicolumn{2}{|c|}{ August 2020 } & $2.28 \pm 2.175$ & 1.50 & $0.00,12.00$ & $1.00,3.00$ \\
\hline \multirow[t]{5}{*}{ Grade $(\%$, No) } & \multirow[t]{2}{*}{ June } & None & \multicolumn{4}{|c|}{$96.2 \%(154)$} \\
\hline & & Mild & \multicolumn{4}{|c|}{$3.8 \%(6)$} \\
\hline & \multirow[t]{3}{*}{ August } & None & \multicolumn{4}{|c|}{$85.0 \%$ (136) } \\
\hline & & Mild & \multicolumn{4}{|c|}{$14.4 \%(23)$} \\
\hline & & Moderate & \multicolumn{4}{|c|}{$0.6 \%(1)$} \\
\hline
\end{tabular}

Table (4): Correlation between TSH and other studied variables.

\begin{tabular}{|l|c|c|}
\hline \multicolumn{1}{|c|}{ All patients $(\mathbf{n = 1 6 0})$} & Correlation coefficient(r) & p \\
\hline Age & -0.065 & 0.413 \\
\hline Duration & -0.147 & 0.064 \\
\hline Dose of levothyroxine & 0.007 & 0.926 \\
\hline Female gender & -0.015 & 0.850 \\
\hline Rural residence & 0.014 & 0.861 \\
\hline Body weight & 0.181 & $\mathbf{0 . 0 2 2} *$ \\
\hline PHQ.9 score at June 2020 & 0.699 & $<\mathbf{0 . 0 0 1} *$ \\
\hline PHQ.9 score at August 2020 & 0.940 & $<\mathbf{0 . 0 0 1} *$ \\
\hline Depression grade at June 2020 & 0.441 & $<\mathbf{0 . 0 0 1} *$ \\
\hline Depression grade at August 2020 & 0.781 & $<\mathbf{0 . 0 0 1} *$ \\
\hline
\end{tabular}

*statistically significant $(\mathrm{p}<0.05)$

\section{8 patients with overt hypothyroidism}

Excluded patients:

- Irregular follow-up visit: 16

- Poor thyroid function monitoring: 10

- Poor adherence: 6

- Prior depression: 2

- COVID-19 infection: 4

\section{Eligible patients: 160}

Figure (1): Flowchart of the study population.

Caption: 198 patients with pre-existing overt hypothyroidism were eligible for inclusion criteria. Based on ongoing application of exclusion criteria, 38 patients were excluded during the study for different reasons. 160 patients continued the study to the end date. 


\section{DISCUSSION}

In the current study, Among 160 patients with a mean age of 42.34 years and $70.6 \%$ female proportion, we noticed progressively increased prevalence of mild depression among Egyptian patients with hypothyroidism during the COVID19 related quarantine. Unfortunately, limited data about the psychological changes of hypothyroid patients during the COVID-19 related lockdown exist. Syed et al. [17] conducted an online questionnaire to screen the prevalence of anxiety and depression among the general public during lockdown of COVID-19. Depression was prevalent in more than half of the participants. Similarly, a multi-waive online study carried out by Hyland et al. [18] to detect the prevalence of anxiety and depression among the Irish people during the COVID-19 pandemic. Approximately, twenty percent of the screened people experienced depression. In China, the psychological distress and depression resulted from COVID-19 related quarantine among the Chinese people was studied by Qiu et al. [19]. They reported psychological distress in about $35 \%$ of the studied population, particularly in females with high education level and close contact to social media as well as the elderly lone people. They attributed detrimental consequences of the quarantine to the augmented fear of infection, death and subsequent socioeconomic burdens. In the current study, we noticed progressively increased prevalence of mild depression during the lockdown of COVID19. Approximately, $15 \%$ of the participants exhibited mild depression in the last follow-up visit which is lower than the aforementioned studies. This inconsistency may be explained by the variation in the study population, design, ethnicity and research methodology. Wang et al. [20] carried out a longitudinal survey at the beginning of COVID-19 outbreak and one month later in 190 different cities in China. Initially, they found a similar rate of depression; approximately $16.5 \%$, with subsequent improvement. In contrary, our patients exhibited progressively increased prevalence of mild depression with continuity of the lockdown. Variation of the study population and period could explain this inconsistency. During the initial period of COVID-19 pandemic, Peng et al. [21] reported depression in $6.21 \%$ among the quarantined people during the lockdown period via the Self-Rating Depression Scale (SDS). On the other side, our participants experienced a higher rate; $15 \%$ over 5 months of the lockdown via PHQ-9 scale.

In the current study, although TSH level remained within the normal reference range, there was a slight progressive rise of TSH level in the latest follow-up visits; June and August $2020 ; 2.54 \pm 1.313$ and $2.73 \pm 1.425$ compared to the former visits; December 2019 and March $2020 ; \quad 2.38 \pm 1.216 \quad$ and $\quad 2.37 \pm 1.212$, respectively. These slight changes may be explained by the strict adherence and commitment of our participants. TSH changes were clinically valueless but were statistically significant. Our results disagreed with Giulia et al. [22] who reported a significant reduction of TSH two months after the lockdown; $1.7 \pm 0.7$ compared to the pre-lockdown values; $2.3 \pm 1.2$ even with definite normal values of TSH in both time points. They attributed TSH reduction to the subsequent changes of work habits, lack of social contacts and the high sensitivity of the hypothalamic pituitary thyroid (HPT) axis to acute stressful situation as was observed by Spaggiari et al. [23] after Italian earthquake swarm.

On the other side, our results are consistent with Fischer S et al. [24] who reported a significant rise of TSH after exposure to acute psychosocial stress. Also, Jung et al. [25] found a strong association between post-traumatic stress disorders and hypothyroidism in female. We found a strong positive correlation between TSH and each of PHQ-9 score and grade of depression throughout the sequential visits during the lockdown. Our results are consistent with Pawel et al. [26] who reported a strong positive correlation between Hashimoto's thyroiditis and score of PHQ-9 scale $(\mathrm{r}=0.767, \mathrm{p}<0.001)$ along with increased risk of depression $(\mathrm{OR}=$ 2.518; $\mathrm{p}<0.001)$ during COVID-19 pandemic. They noticed that patients with Hashimoto's thyroiditis experienced more frequent and sever symptoms of depression than patients with other chronic diseases during the pandemic.

We faced many limitations such as the small sample due to dropout of eligible patients, fear of hospital attendance, fear of contact with laboratory, lack of a control group of healthy subjects without hypothyroidism or hypothyroid subjects out of the lockdown measures and lack of thyroid autoantibodies assessment which may potentially surge with stress particularly among those with prior diagnosed Hashimoto's 
thyroiditis. Multi-center trials with a larger scale, multiple ethnicities are needed in the future. The strength points of this study included selection of properly compensated hypothyroid patients with strict adherence to levothyroxine therapy, the longitudinal follow-up design to get a real-world evidence of the alterations of thyroid function and depression during the lockdown, and finally excluding any patient with prior depression or COVID-19 infection that may disrupt the psychological and endocrinal homeostasis [27].

\section{CONCLUSION}

Throughout the period of COVID-19 related quarantine, Egyptian patients with pre-existing hypothyroidism experienced progressively increased prevalence of mild depression..

Abbreviations: Coronavirus disease 2019 (COVID-19), Patient Health Questionnaire-9 (PHQ-9), severe acute respiratory syndrome coronavirus 2 (SARS-CoV-2), Thyroid stimulating hormone (TSH), the Self-Rating Depression Scale (SDS), the hypothalamic pituitary thyroid (HPT).

Funding: by authors

Conflict of interest: none Ethical standards:

An official approval was gained from of the Institutional Research Board (IRB) of scientific research committee in Mansoura University with the approval number (R.21.02.1221.R1). All procedures were in accordance with the ethical standards of the institutional research committee and with the 1975 Helsinki Declaration and its later amendments or comparable ethical standards. Written informed consent was approved by the IRB and signed by all participants.

\section{Highlights:}

1- Psychological burdens of COVID-19 were extensively studied on the general population. However, the impact of the lockdown on thyroid function and depression in Egyptian patients with hypothyroidism needs more clarification. 2- We excluded any patient with prior or current, proved or suspected COVID-19 exposure to rule out the confounding effect of SARS-CoV-2 on thyroid function and psychological status.
3- Initially, we included patients who exhibited proper commitment and adherence to thyroid replacement therapy to avoid the confounding effect of disrupted thyroid function on psychological status outcomes. 4- This longitudinal follow-up study was conducted throughout the lockdown period over multiple stations with a potential novel design in this category of our patients to get real-world evidence of the proposed target. 5- The results of this study raised a significant warning about the probable increased risk of depression among hypothyroid patients even with minimal changes of TSH.

\section{REFERENCES}

1. Abdelmonem Awad Hegazy, Raafat Awad Hegazy.COVID-19: Virology, Pathogensis and Potential Therapeutics. Afro-Egyptian Journal of Infectious and Endemic Diseases. Article 3, Volume 10, Issue 2, June 2020, Page 93-99 DOI: $10.21608 /$ aeji.2020.93432

2. Bao Y., Sun Y., Meng S., Shi J., Lu L. 2019nCoV epidemic: Address mental health care to empower society. Lancet. 2020;395:e37-e38. doi: 10.1016/S0140-6736(20)30309-3. [PMC free article] [PubMed] [CrossRef] [Google Scholar]

3. Louvardi M, Pelekasis P, Chrousos GP, Darviri C. Mental health in chronic disease patients during the COVID-19 quarantine in Greece. Palliat Support Care. 2020 Aug; 18(4):394-399. doi: $10.1017 / \mathrm{S} 1478951520000528$.

4. Shigemura J., Ursano R.J., Morganstein J.C., Kurosawa M., Benedek D.M. Public responses to the novel 2019 coronavirus (2019-nCoV) in Japan: mental health consequences and target populations. Psychiatry Clin. Neurosci. 2020; 74:281-282. doi: 10.1111/pcn.12988. [PMC free article] [PubMed] [CrossRef] [Google Scholar] [Ref list]

5. Wang C, Pan R, Wan X, Tan Y, Xu L, Ho CS, et al. Immediate Psychological Responses and Associated Factors during the Initial Stage of the 2019 Coronavirus Disease (COVID-19) Epidemic among the General Population in China. Int. J. Environ. Res. Publ. Health. 2020; 17(5):1729. doi: 10.3390/ijerph17051729. [PMC free article] [PubMed] [CrossRef] [Google Scholar]

6. Martí O, Gavaldà A, Jolín T, Armario A. Effect of regularity of exposure to chronic immobilization stress on the circadian pattern of pituitary adrenal hormones, growth hormone, and thyroid stimulating hormone in the adult male rat. Psychoneuroendocrinology. 1993; 
18(1):67-77. doi: 10.1016/0306-4530(93)90056q. [PubMed] [CrossRef] [Google Scholar]

7. Helmreich DL, Parfitt DB, Lu XY, Akil H, Watson SJ. Relation between the hypothalamicpituitary-thyroid (HPT) axis and the hypothalamic-pituitary-adrenal (HPA) axis during repeated stress. Neuroendocrinology. 2005; 81(3): 183-192. doi: 10.1159/000087001. [PubMed] [CrossRef] [Google Scholar]

8. Asfandyar Khan Niazi, Sanjay Kalra, Awais Irfan, Aliya Islam. Thyroidology over the ages. Indian J. Endocrinol. Metab. 2011; 15 (Suppl 2):S121-S126. doi: 10.4103/2230-8210.83347. [PMC free article] [PubMed] [CrossRef] [Google Scholar]

9. Rouchell A.M., Pouns R., Tierney J.G. Depression. In: Wise M.G., Rundell J.R., editors. Textbook of consultation-liaison psychiatry. Psychiatry in the medically ill. 2nd ed. American Psychiatric Publishing; Washington, DC: 2002. pp. 307-338. [Google Scholar]

10. Degner D., Meller J., Bleich S., Schlautmann V., Rüther E. Affective Disorders Associated With Autoimmune Thyroiditis. J. Neuropsychiatry Clin. Neurosci. 2001; 13:532-533. doi: 10.1176/jnp.13.4.532. [PubMed] [CrossRef] [Google Scholar]

11. Carta M.G., Hardoy M.C., Carpiniello B., Murru A., Marci A.R., Carbone F., et al. A case control study on psychiatric disorders in Hashimoto disease and Euthyroid Goitre: Not only depressive but also anxiety disorders are associated with thyroid autoimmunity. Clin Pract. Epidemiol. Ment Health. 2005; 1:23. doi: 10.1186/1745-0179-1-23. [PMC free article] [PubMed] [CrossRef] [Google Scholar]

12. Giynas Ayhan M., Uguz F., Askin R., Gonen M.S. The prevalence of depression and anxiety disorders in patients with euthyroid Hashimoto's thyroiditis: A comparative study. Gen. Hosp. Psychiatry. 2014; 36:95-98. doi: 10.1016/j.genhosppsych.2013.10.002. [PubMed] [CrossRef] [Google Scholar]

13. Kirim S., Keskek S.O., Köksal F., Haydardedeoglu F.E., Bozkirli E., Toledano Y. Depression in patients with euthyroid chronic autoimmune thyroiditis. Endocr. J. 2012; 59:705-708. doi: 10.1507/endocrj.EJ12-0035. [PubMed] [CrossRef] [Google Scholar]

14. Spencer CA, LoPresti JS, Patel A, Guttler RB, Eigen A, Shen D, et al. Applications of a new chemiluminometric thyrotropin assay to subnormal measurement. J. Clin. Endocrinol. Metab. 1990; 70(2):453-460. doi: 10.1210/jcem-70-2-453. [PubMed] [CrossRef] [Google Scholar]

15. Shashank R Joshi. Laboratory evaluation of thyroid function. J Assoc Physicians India. 2011 Jan; 59 Suppl:14-20.

16. Kroenke K, Spitzer RL, Williams JB. The PHQ-9: validity of a brief depression severity measure. J Gen Intern Med. 2001 Sep;16(9):60613. doi: 10.1046/j.1525-1497.2001.016009606.x.

17. Syed Mustafa Ali Shah, Danish Mohammad, Muhammad Fazal Hussain Qureshi, Mohammad Zain Abbas, Sameeha Aleem Prevalence, Psychological Responses and Associated Correlates of Depression, Anxiety and Stress in a Global Population, During the Coronavirus Disease (COVID-19) Pandemic Community Ment Health J. 2020 Oct 27 : 1-10.doi: 10.1007/s10597-020-00728-y

18. P Hyland, M Shevlin, O McBride, J Murphy, T Karatzias, R P Bentall, et al, .Anxiety and depression in the Republic of Ireland during the COVID-19 pandemic. Acta Psychiatr Scand. 2020 Sep; 142(3):249-256. doi: 10.1111/acps.13219. Epub 2020 Aug 9.

19. Qiu J, Shen B, Zhao M, Wang Z, Xie B, Xu Y. A nationwide survey of psychological distress among Chinese people in the COVID-19 epidemic: implications and policy recommendations. Gen Psychiatr. 2020; 33(2): e100213.Published online 2020 Mar 6. doi: 10.1136/gpsych-2020-100213

20. Wang C, Pan R, Wan X, Tan Y, Xu L, McIntyre R.S, et al. A longitudinal study on the mental health of general population during the COVID-19 epidemic in China. Brain Behav Immun. $2020 \mathrm{Jul}$; 87: 40-48.Published online 2020 Apr 13. doi: 10.1016/j.bbi.2020.04.028

21. Peng M, Mo B, Liu Y, Xu M, Song X, Liu L, et al. Prevalence, risk factors and clinical correlates of depression in quarantined population during the COVID-19 outbreak. J Affect Disord. 2020 Oct 1; 275: 119124.Published online 2020 Jul 2. doi: 10.1016/j.jad.2020.06.035

22. Giulia Brigante, Giorgia Spaggiari, Barbara Rossi, Antonio Granata, Manuela Simoni, and Daniele Santi . A prospective, observational clinical trial on the impact of COVID-19-related national lockdown on thyroid hormone in young males. Sci Rep. 2021; 11: 7075. Published online 2021 Mar 29. doi: 10.1038/s41598-02186670-9

23. Spaggiari G, Setti M, Tagliavini S, Roli L, De Santis MC, Trenti T, et al. The hypothalamicpituitary-adrenal and -thyroid axes activation lasting one year after an earthquake swarm: 
results from a big data analysis. J. Endocrinol. Invest. 2020 doi: 10.1007/s40618-020-01457-5. [PubMed] [CrossRef] [Google Scholar]

24. Fischer S, Strahler J, Markert C, Skoluda N, Doerr JM, Kappert M, et al. Effects of acute psychosocial stress on the hypothalamicpituitary-thyroid (HPT) axis in healthy women. Psychoneuroendocrinology. 2019; 110:104438. doi: 10.1016/j.psyneuen.2019.104438. [PubMed] [CrossRef] [Google Scholar]

25. Jung SJ, Kang JH, Roberts AL, Nishimi K, Chen Q, Sumner JA, et al. Posttraumatic stress disorder and incidence of thyroid dysfunction in women. Psychol. Med. 2019; 49(15):2551-2560. doi: 10.1017/s0033291718003495. [PubMed] [CrossRef] [Google Scholar]

26. Paweł Wańkowicz, Aleksandra Szylińska, and Iwona Rotter. The Impact of the COVID-19 Pandemic on Psychological Health and Insomnia among People with Chronic DiseasesJ Clin Med. 2021 Mar; 10(6): 1206. Published online 2021 Mar 14. doi: 10.3390/jcm10061206

27. Khoo B, Tan T, Clarke SA, Mills EG, Patel B, Modi M, et al. Thyroid Function Before, During, and After COVID-19. J. Clin. Endocrinol. Metab. 2021; 106(2):e803-e811. doi: 10.1210/clinem/dgaa830. [PMC free article] [PubMed] [CrossRef] [Google Scholar]. 